\title{
Associations between self-reported and objectively measured physical activity, sedentary behavior and overweight/obesity in NHANES 2003-2006
}

\author{
Wanner, Miriam ; Richard, Aline ; Martin, Brian ; Faeh, David ; Rohrmann, Sabine
}

\begin{abstract}
OBJECTIVE: To investigate associations between self-reported and objectively measured physical activity, sedentary behavior and overweight/obesity based on percent body fat measured with Dual Energy X-Ray Absorptiometry (DXA), waist circumference (WC), waist-to-height ratio and body mass index, focusing on different intensities and domains of physical activity. METHODS: Data from NHANES 2003-2006 were analyzed using linear and ordered logistic regression analyses. A total of 4794 individuals aged 18-69 years with valid physical activity and DXA data were included. Objectively measured physical activity and sedentary behavior were assessed using accelerometers, self-reported physical activity using the NHANES physical activity questionnaire. Weight, height, WC and DXA measures were assessed in the mobile examination centers. RESULTS: We observed statistically significant associations between objectively measured moderate and vigorous physical activity and all definitions of overweight/obesity. For total physical activity, the odds of being in the higher percent body fat category were $0.56(95 \%$ confidence interval (CI) $0.41,0.77)$ for the medium and 0.30 (95\% CI $0.22,0.40)$ for the highest physical activity tertile compared with the lowest. For light activities, lifestyle activities and sedentary behavior, associations were only observed in the linear models with percent total body fat but not in the ordered logistic regression models. Regarding self-reported physical activity, consistent significant associations with overweight/obesity were only observed for vigorous and for transport activity. CONCLUSIONS: Regarding moderate and vigorous physical activity, more active individuals were less affected by overweight/obesity than less active individuals, emphasizing the public health effect of physical activity in the prevention of overweight/obesity. The fact that associations were more consistent for objectively measured than for self-reported physical activity may be due to bias related to self-reporting. Associations between lower intensity activities and overweight/obesity were weak or inexistent.International Journal of Obesity advance online publication, 25 October 2016; doi:10.1038/ijo.2016.168.
\end{abstract}

DOI: https://doi.org/10.1038/ijo.2016.168

Posted at the Zurich Open Repository and Archive, University of Zurich

ZORA URL: https://doi.org/10.5167/uzh-128007

Journal Article

Accepted Version

Originally published at:

Wanner, Miriam; Richard, Aline; Martin, Brian; Faeh, David; Rohrmann, Sabine (2017). Associations between self-reported and objectively measured physical activity, sedentary behavior and overweight/obesity in NHANES 2003-2006. International Journal of Obesity, 41(1):186-193.

DOI: https://doi.org/10.1038/ijo.2016.168 
$4 \quad$ Running title: Physical activity, sedentary behavior and obesity

5

6 Miriam Wanner $^{1,2)}$, Aline Richard ${ }^{1)}$, Brian Martin ${ }^{1,3)}$, David Faeh $^{1,4)}$, Sabine Rohrmann ${ }^{1,2)}$

7

$8 \quad{ }^{1)}$ Epidemiology, Biostatistics and Prevention Institute, University of Zurich, Switzerland

$9 \quad{ }^{2)}$ Cancer Registry Zurich and Zug, University Hospital Zurich, Switzerland

${ }^{3)}$ Office of Public Health, Canton of Basel-Country, Liestal, Switzerland

4) Health Division, Department of Business, Health and Social Work, Bern University of Applied

Sciences, Bern, Switzerland

13

Corresponding author:

Miriam Wanner, PhD

University of Zurich

Epidemiology, Biostatistics and Prevention Institute

Hirschengraben 84

8001 Zurich

Switzerland

Phone: +41 442551716

Fax: +41442555636

Email: miriam.wanner@uzh.ch

\section{Conflict of interest}

The authors declare no conflict of interest. 
To investigate associations between self-reported and objectively measured physical activity, sedentary behavior and overweight/obesity based on percent body fat measured with Dual Energy XRay Absorptiometry (DXA), waist circumference (WC), waist-to-height ratio (WHtR) and body mass index (BMI), focusing on different intensities and domains of physical activity.

Methods

Data from NHANES 2003-2006 were analyzed using linear and ordered logistic regression analyses.

A total of 4794 individuals aged 18-69 years with valid physical activity and DXA data were included.

Objectively measured physical activity and sedentary behavior were assessed using accelerometers, self-reported physical activity using the NHANES physical activity questionnaire. Weight, height, WC and DXA measures were assessed in the mobile examination centers.

Results

We observed statistically significant associations between objectively measured moderate and vigorous physical activity and all definitions of overweight/obesity. For total physical activity, the odds of being in the higher percent body fat category were 0.56 (95\% CI $0.41,0.77$ ) for the medium and $0.30(95 \%$ CI $0.22,0.40)$ for the highest physical activity tertile compared to the lowest. For light activities, lifestyle activities and sedentary behavior, associations were only observed in the linear models with percent total body fat but not in the ordered logistic regression models. Regarding selfreported physical activity, consistent significant associations with overweight/obesity were only observed for vigorous and for transport activity.

\section{Conclusions}

Regarding moderate and vigorous physical activity, more active individuals were less affected by overweight/obesity than less active individuals, emphasizing the public health effect of physical activity in the prevention of overweight/obesity. The fact that associations were more consistent for objectively measured than for self-reported physical activity may be due to bias related to selfreporting. Associations between lower intensity activities and overweight/obesity were weak or inexistent. 


\section{Introduction}

Physical activity contributes up to $50 \%$ of total daily energy expenditure, ${ }^{1}$ and there is evidence that active individuals have a healthier body weight and composition than inactive individuals. ${ }^{2-4}$ To date, the majority of evidence on associations between physical activity, sedentary behavior and overweight/obesity has been derived from self-report measures, with body mass index (BMI) as most frequently used measure for the definition of overweight/obesity. However, there is controversy on whether BMI is appropriate for defining people at risk. ${ }^{5}$ Measures of central obesity like waist circumference (WC), ${ }^{6}$ waist-to-hip ratio $(\mathrm{WHR})^{6,7}$ and waist-to-height ratio $(\mathrm{WHtR})^{5}$ were better discriminators for morbidity and mortality risk. Moreover, Dual Energy X-Ray Absorptiometry (DXA) is considered a gold-standard measurement of body composition, ${ }^{8}$ however, not many studies include such measures due to resource limitations.

Only few studies have investigated the association between objectively measured physical activity and sedentary behavior and overweight/obesity, mostly in small samples ${ }^{9-11}$ and specific sub groups. ${ }^{10,11}$ Some publications are available based on the Health Survey for England mostly regarding associations between sedentary behavior (both self-reported and accelerometer-derived) ${ }^{12,13}$ but also physical activity $^{14}$ and cardiometabolic risk factors including BMI.

Earlier studies using NHANES data reported associations between objectively measured physical activity and overweight, ${ }^{15}$ or both sedentary time and physical activity on overweight. ${ }^{16,17}$

Most evidence on associations between physical activity and weight status is available for leisure-time physical activity, ${ }^{18}$ whereas less is known about the impact of other domains of physical activity. Furthermore, to our knowledge, there is no study including both objective measures of physical activity and sedentary behavior and percent body fat based on DXA measurements.

The National Health and Nutrition Examination Survey (NHANES) 2003-06 provides information on objectively measured (accelerometers) and self-reported (questionnaire) physical activity as well as various measures of body composition. The aim of this study was to investigate cross-sectional associations between self-reported and objectively measured physical activity and sedentary behavior on the one hand and different anthropometric measures and derived overweight/obesity definitions on 
the other hand. Regarding physical activity, we focused on different intensities (light, lifestyle, moderate and vigorous) and different domains (leisure-time, transport, household).

\section{Materials and Methods}

Sample

We used data from NHANES 2003-2006, representative of the US civilian non-institutionalized population. A multistage probability sampling was used to recruit participants. The NCHS Institutional Review Board has reviewed and approved the study protocol and all participants gave written informed consent. ${ }^{19}$

We considered 9173 individuals aged 18-69 years (DXA was only measured in individuals aged $\leq 69$ years). Of these, we excluded 390 individuals because they only participated in the interview, 694 because of missing DXA data and 416 due to missing data on dietary intake (N=7673). Furthermore, 2801 individuals were excluded because they had not sufficient valid objectively measured physical activity data and two individuals due to missing self-reported physical activity data ( $\mathrm{N}=4870)$. Finally, 76 individuals were excluded because they were categorized as underweight, leaving 4794 individuals for analyses. For sensitivity analyses, we repeated the analyses for self-reported physical activity without excluding individuals that had missing objectively measured physical activity.

\section{Measures}

Physical activity

Physical activity was assessed objectively in 2003/04 and 2005/06 using the validated ActiGraph 7164 accelerometer (ActiGraph, Shalimar, FL) ${ }^{20}$ The participants were instructed to wear an accelerometer placed on their right hip for 7 consecutive days following the examination at the medical examination center (MEC). The chosen epoch time was 1 minute. Inclusion criteria for the present analyses were: $\geq 4$ days of data with $\geq 10$ hours per day of accelerometer wear time. ${ }^{21}$

We used the following cut points for quantifying the duration of physical activity in different intensity levels: sedentary $\left(<100\right.$ counts/min), ${ }^{22}$ light $\left(100-759\right.$ counts/min), lifestyle (760-2019 counts/min), ${ }^{23}$ moderate (2020-5998 counts/min) and vigorous ( $\geq 5999$ counts/min) ${ }^{24}$ Counts/minute, minutes spent 
sedentary, in light, lifestyle, moderate and moderate-to-vigorous physical activity per day as exposure variables were categorized into tertiles. Minutes spent in vigorous physical activity were only categorized into two groups (low=none, high=any) because $60 \%$ of the participants had no vigorous activities.

Self-reported physical activity was derived from the NHANES physical activity questionnaire. METminutes per week spent in different domains of physical activity (leisure-time, household, transport) were calculated and categorized into tertiles, except for transport activity which was dichotomized because $70 \%$ of participants reported no such activity. Minutes per week of moderate and vigorous activities were calculated. Because $64 \%$ of participants reported no vigorous activity, this variable was dichotomized into low (=none) and high (=any). For moderate activities, $44 \%$ reported no such activities, these were categorized as low. The remaining $56 \%$ were distributed evenly into the categories medium and high.

Overweight/obesity

DXA measurements

The whole body DXA scans were conducted using a Hologic QDR 4500A fan-beam densitometer (Hologic, Inc., Bedford, Massachusetts) following the manufacturer's acquisition procedures. Exclusion criteria during the examination were pregnancy and self-reported weight $>136 \mathrm{~kg}$ or height $>196 \mathrm{~cm}$, exceeding the DXA table limits. The scan for each survey participant was reviewed, for details see the "Technical Documentation for the 1999-2004 Dual Energy X-Ray Absorptiometry (DXA) Multiple Imputation Data files (2008). ${ }^{25}$

A DXA scan provides multiple data points for each individual. The amount of missing DXA data was larger than for other type of data with indication of systematic, non-random patterns. ${ }^{25}$ Because this could bias the results, users are provided with a "complete" data file, missing and invalid DXA data were imputed ("filling in plausible values for missing data") using multiple-imputation methodology. As a result, each survey participant gets five complete records containing valid (measured) and/or imputed values. For those with multiply imputed data, the five records consist of different sets of 
imputed values. Participants with fully valid data have 5 identical records because no imputation was

141

necessary, for details see ${ }^{25}$.

Android (more "risky" central fat accumulation: "male" or "apple" shape) and gynoid (more "protective" peripheral fat accumulation: "female" or "pear" shape) fat distributions ${ }^{26}$ were defined by the integrated Hologic APEX software. Percent total body fat derived from DXA measurement was categorized as normal weight $(<25 \%$ for men, $<35 \%$ for women) and overweight/obese $(\geq 25 \%$ for men, $\geq 35 \%$ for women $)^{27}$

\section{Other measures of overweight and obesity}

As secondary outcomes, BMI, WC, and WHtR were included. Weight was measured using a Toledo digital scale with the participants wearing underwear only. ${ }^{28}$ Standing height was measured with a fixed stadiometer with a vertical backboard and a moveable headboard. ${ }^{28}$ BMI was calculated as weight (in $\mathrm{kg}$ ) divided by height squared (in meters) and categorized as normal weight (18.5-25 $\mathrm{kg} / \mathrm{m} 2)$, overweight $(25-<30 \mathrm{~kg} / \mathrm{m} 2)$, and obese $(\geq 30 \mathrm{~kg} / \mathrm{m} 2) .{ }^{29} \mathrm{WC}$ was measured by trained health technicians in a standing position just above the uppermost lateral border of the ilium at the end of a normal expiration. ${ }^{28}$ It was categorized as normal weight $(<94 \mathrm{~cm}$ for men, $<80 \mathrm{~cm}$ for women), overweight $(94-<102 \mathrm{~cm} / 80-<88 \mathrm{~cm})$, and obese $(\geq 102 \mathrm{~cm} / \geq 88 \mathrm{~cm}){ }^{29-31}$ Finally, WHtR was categorized as normal weight $(<0.5)$, overweight $(0.5-<0.6)$, and obese $(\geq 0.6) .^{32,33}$

\section{Other variables}

Socio-demographic variables (sex, age, race/ethnicity, education, marital status, and poverty income ratio $[P I R]$ ) and health-related variables (smoking status, alcohol intake, general health status, and mean energy intake) were included as potential confounders. Ethnicity was categorized as nonHispanic White, non-Hispanic Black, Mexican-American, and other. Education was dichotomized into post-secondary education (college or higher) versus lower. Marital status was defined as living together versus single (including widowed, separated, divorced and never married). PIR was included as a measure of socio-economic status (at or above poverty versus below poverty). 
Self-reported smoking behavior (current smoker versus current non-smoker), alcohol intake (no drinks, $\leq 1$ drink per week, and $>1$ drink per week), sedentary behavior (mean minutes per day based on accelerometer data), and mean energy intake (kilo calories based on $24 \mathrm{~h}$ recalls) were used as general healthy living indicators. Finally, general health status was included based on questionnaire data (excellent/very good, good, and not so good/poor). The dietary data to calculate total energy intake was based on the mean of two separate $24 \mathrm{~h}$ recall interviews (if available, otherwise based on day 1), the first one conducted in-person during the MEC examination, the second one three to ten days later by telephone.

\section{Statistical analyses}

For descriptive statistics, weighted means and standard deviations for continuous variables and proportions for categorical variables are presented. For the DXA measures, the 5 sets of imputations were used according to the recommendations of NHANES ${ }^{25}$ in order to correct for missing data, which are not at random. The five estimates were used to generate an overall mean, and combined standard errors were used to derive the overall $95 \%$ confidence intervals based on ${ }^{25}$.

Ordered logistic regression models were used to model the association between different measures of physical activity and sedentary behavior on overweight/obesity as "ordered" outcome. Separate models were run for each physical activity variable with each overweight/obesity variable. Sexspecific tertiles of the physical activity and sedentary behavior variables were used as exposure with "low" as the reference category (versus medium and high). Because for vigorous and transport activities more than $50 \%$ of the participants had no such activities, we used only two categories (none, any). Linear regression analyses were used to investigate associations between continuous physical activity variables and percent body fat (total, android, gynoid, each as continuous variable in a separate model). These analyses were stratified by sex because of the sex-specific distribution (android = "male" or "apple" shape, gynoid = "female" or "pear" shape).

All analyses were adjusted for potential confounders (sociodemographic variables, health-related behavior). Additionally, the models that included a physical activity variable were adjusted for 
sedentary behavior (but not for other physical activity variables due to collinearity), the models including sedentary behavior were adjusted for moderate-to-vigorous physical activity.

Four categories based on moderate-to-vigorous physical activity (MVPA, accelerometer) and sedentary behavior (accelerometer) were defined by splitting the two variables at their median: low MVPA/high sedentary time (reference), low MVPA/low sedentary time, high MVPA/high sedentary time, and high MVPA/low sedentary time. Associations of these variables with different measures of overweight and obesity were assessed using ordered logistic regression analyses.

All analyses were weighted according to the NHANES guidelines. ${ }^{19}$ Statistical significance was set at $\mathrm{P}<0.05$. The analyses were performed using STATA version 12 (Stata Corporation, College Station, TX, USA). Code availability: Please contact the corresponding author.

\section{Results}

Mean age of the study participants was 44.3 years and the proportion of women was $50.6 \%$. Table 1 displays the characteristics of the participants by sex and body fat category. Overweight/obese men and women tended to be older compared to normal weight individuals, respectively.

Total, android and gynoid percent body fat and WHtR were significantly higher in women than men. WC was significantly higher in men (no significant sex difference for BMI). Men were more likely to be overweight according to BMI and WHtR, women according to percent body fat. Women were more likely than men to be obese according to WC and WHtR.

Physical activity levels tended to be lower in overweight/obese individuals and in women according to both objectively measured and self-reported instruments. Sedentary behavior was higher in overweight/obese individuals. Total energy intake was lower in women and overweight/obese individuals. Normal weight individuals and men more likely drank $\geq 1$ alcoholic drink per week and smoked currently. Normal weight individuals more likely reported excellent health status compared to overweight individuals.

Table 2 shows the results of the ordered logistic regression analyses for categories of percent body fat, BMI, WC and WHtR associated with different objectively measured physical activity variables. 
Statistically significant inverse associations were observed for mean counts/min, moderate, vigorous, and moderate-to-vigorous physical activity with all four overweight/obesity parameters. For example, the odds of being in a higher BMI category (i.e. overweight versus normal weight and obese versus overweight) was 0.64 (95\% CI $0.50,0.82)$ for individuals in the medium tertile and 0.40 (95\% CI $0.30,0.52)$ for those in the top tertile compared to those in the bottom tertile of moderate-to-vigorous physical activity. No associations were present for lifestyle activities, light activities and for sedentary behavior.

Table 3 shows the respective results for self-reported physical activity. Associations were most consistent for vigorous physical activity and for transport activities with all overweight/obesity outcomes. In addition, there were statistically significant associations between the top tertile of total (moderate-to-vigorous) physical activity and percent body fat, WC and WHtR, and between the top tertile of total leisure-time physical activity and percent body fat, WC and WHtR. For household physical activity, more physical activity was associated with higher odds of being in higher overweight/obesity categories. When including individuals with missing objectively measured physical activity in these analyses (sensitivity analysis), the results did not change substantially (for example, OR for being in a higher percent body fat category when in the highest tertile of total selfreported physical activity [compared to the lowest tertile]: $\mathrm{OR}=0.47$ [95\% CI $0.35,0.62$ ] for both samples in the fully adjusted model).

According to Supplementary Information Table 1 (linear regression models), there were statistically significant inverse associations between the objectively measured physical activity variables and percent total body fat such that more physical activity was associated with lower percentage of body fat. For example, total body fat was on average $0.21 \%$ (95\% CI $0.17,0.25)$ lower for each additional 100 MET-min/week of moderate-to-vigorous physical activity. In general, the associations were stronger for women than for men and slightly stronger for android than for gynoid fat areas. For light activities, the associations were significant for total and android fat in the non-stratified analyses. Unlike in the ordered logistic regression models (Table 2), there was an association between sedentary behavior and percent body fat (i.e., for each additional hour of sedentary time per day percent total body fat was on average $0.16 \%$ [95\% CI $0.02,0.30]$ higher); however, this association was only 
present for total body fat but not for android or gynoid fat or stratified by sex. For self-reported

251

252

253

254

255

256

257 physical activity, the associations were generally weaker and consistently statistically significant only for total leisure-time physical activity and for vigorous physical activity.

Figure 1 shows the associations between the four categories of low and high objectively measured moderate-to-vigorous physical activity/sedentary behavior and different measures of overweight/obesity. The distribution of participants in the four categories was $29.7 \%$ for low MVPA/high sedentary time, 20.6\% for low MVPA/low sedentary time, 20.3\% for high MVPA/high sedentary time and $29.4 \%$ for high MVPA/low sedentary time. The odds of being in a higher category of percent body fat, BMI, WC and WHtR were statistically significantly lower for individuals with high objectively measured moderate-to-vigorous physical activity independent of sedentary behavior, whereas low compared to high sedentary time was not significantly associated with body weight status.

\section{Discussion}

We observed statistically significant associations between objectively measured moderate, vigorous and total physical activity and all measures of overweight/obesity (BMI, percent body fat based on DXA measures, WC, and WHtR). Associations for light and lifestyle physical activity and for sedentary behavior were weak or non-significant and were only observed in the non-stratified linear models. Regarding self-reported physical activity, consistent significant associations with all overweight/obesity variables were observed for vigorous physical activity and transport activity (ordered logistic regression), and for total and vigorous physical activity (linear regression). When looking at the combined association of moderate-to-vigorous physical activity and sedentary behavior (Figure 1), it seems that sedentary behavior is not associated with overweight/obesity independent of the physical activity category supporting our null findings for sedentary behavior in Table 2 .

For objectively measured physical activity, we observed consistent associations with overweight/obesity both for moderate and vigorous physical activity, but for self-reported physical activity associations were only present for vigorous but not for moderate activities. One possible 
explanation is that the intensity of the activity was overestimated and, thus, self-reported moderate physical activity corresponds better with accelerometer-derived light activities. This is supported by a validation study, which indeed indicated that self-reported moderate activities correlate better with objectively measured light than with moderate activities. ${ }^{34}$ In addition, the fact that objectively measured physical activity was more consistently associated with overweight/obesity than selfreported physical activity indicates that the latter may be more prone to misclassification, especially regarding moderate physical activity and household tasks. On the other hand, the advantage of the self-reported physical activity data is that information regarding domains (leisure-time, transport, household) is available.

Regarding overweight/obesity, the associations were generally strongest for the objective assessment of body composition (DXA based) and weakest for BMI. For example, self-reported physical activity associations were more consistent for percent body fat and for WHtR than for BMI and WC (Table 3). Similar results have been reported in a Swiss study on self-reported physical activity based on the International Physical Activity Questionnaire IPAQ and different measures of overweight and obesity. ${ }^{35}$ A reason may be that percent body fat and WHtR are more appropriate measures to define overweight/obesity than, for example, BMI, and that associations become diluted if measurement error in self-reported physical activity is present. For example, BMI is influenced by body fat mass, but also by muscle mass, which are both associated with physical activity. ${ }^{36}$

Based on linear regression models (Supplementary Information Table 1), the strength of association between physical activity and percent body fat decreases with decreasing intensity, indicating that higher intensity activities may be more effective in weight management, even though it seems that also lower-intensity activities have at least some small effect. Similarly, but also taking into account duration in addition to intensity, a dose-response association between total amounts of self-reported leisure-time physical activity and obesity has been reported in adult women, but not in men. ${ }^{37}$ In our study, the effect sizes were generally slightly stronger for android than for gynoid fat (Supplementary Information Table 1) and for women compared to men.

The results of other studies investigating the associations between objectively measured physical activity and sedentary time with overweight/obesity were mixed. Based on NHANES data from 2003- 
04, Strath et al. reported associations both for bout and non-bout moderate-to-vigorous physical activity with BMI and WC, with stronger effects for activity performed in bouts of at least 10 minutes. ${ }^{15}$ Even though in our analyses adjustment for sedentary behavior did not change the direction of the results, it has to be mentioned that the analyses by Strath et al. were not adjusted for sedentary behavior and for diet. Healy et al. focused on the effect of sedentary time and breaks in sedentary time (based on accelerometry) on WC (among other biomarkers) using NHANES data from 2003-2006. ${ }^{16}$ Using linear regression models and physical activity and dietary variables as confounders, the authors reported statistically significant associations between sedentary time, breaks in sedentary time and WC. However, when stratifying the analyses by race/ethnicity, the results for sedentary behavior were only statistically significant in the expected direction for non-Hispanic Whites, with null effects for Mexican Americans and effects in the other than expected direction for non-Hispanic Blacks. Again, using NHANES data from 2003-2006, Maher and colleagues investigated the associations between physical activity and sedentary time and obesity based on BMI. ${ }^{17}$ Similar to our results, the authors concluded that physical activity was strongly associated with BMI regardless of sedentary time, whereas no significant results were reported for sedentary time. A number of reports have been published based on the Health Survey for England ${ }^{12-14}$ showing that objectively measured physical activity was associated with BMI, ${ }^{14}$ while there were no associations between objectively measured sedentary time and WC or BMI in working-age adults ${ }^{13}$ and only with WC but not BMI in older adults. ${ }^{12}$ This is again in line with our results.

Regarding associations between different domains of physical activity and overweight/obesity, most other studies reported no favorable (inverse) association for domestic physical activity, ${ }^{38,39}$ which is in line with our results. This may be due to residual confounding or larger reporting bias as individuals may be likely to over-report intensity and/or duration of domestic activities compared to e.g. leisuretime activities. An inverse relationship between transport physical activity and overweight/obesity has also been reported in a systematic review, ${ }^{40}$ and evidence regarding an association between leisuretime physical activity and weight status is also available. ${ }^{18}$

332 Like most others, this is a cross-sectional study and no causal inference is possible. It is commonly assumed that physical activity is a determinant of body fat due to energy expenditure. ${ }^{41}$ However, 
more recent research suggests that obesity is a determinant of physical activity rather than the other way around, in that baseline body fat predicted follow-up physical activity. ${ }^{41}$ On the other hand, physical activity patterns over a ten year period (remaining inactive versus remaining active) were significantly associated with obesity at follow-up in a recent study. ${ }^{35}$ A similar discussion regarding reverse causality is ongoing for sedentary behavior. Research based on large longitudinal studies has pointed out that obesity is a predictor of self-reported sitting time while associations in the other direction were unclear or not significant. ${ }^{42-44}$ It is therefore possible that significant associations between sedentary time and overweight/obesity in cross-sectional studies (see e.g. Healy et al. $\left.(2011)^{16}\right)$ are attributable to reverse causality. More research is needed to disentangle the longitudinal associations between physical activity, sedentary behavior and overweight/obesity ideally using objective measurements.

345 The strengths of this study are the large and US nationally representative sample, the use of objectively measured data for physical activity, sedentary behavior and body composition, the use of self-reported physical activity data regarding domains, and the inclusion of a variety of potential confounders including socio-demographic variables, health-related variables and diet. Limitations of the study are its cross-sectional design and thus the possibility of reverse causality (overweight and obese people may be less likely to be active due to their body mass), the fact that accelerometers had to be taken off during water-based activities and that some activities are not measured well using uniaxial accelerometer data, such as bicycling. This could result in an underestimation of physical activity. Accelerometer wear time that is smaller than mean time awake may be another issue, however mean wear time was $>14$ hours and was therefore relatively high. There is also a limitation of accelerometers in assessing sedentary behavior. Even though we have adjusted for a number of covariates, we cannot rule out the possibility of residual confounding. Regarding self-reported physical activity, recall bias may be a problem.

358 In conclusion, objectively measured physical activity was associated with all overweight/obesity 359 variables, emphasizing the public health effect of physical activity in the prevention of overweight/obesity. Associations between lower intensity activities and overweight/obesity were weak 
361 or inexistent. More research is needed to disentangle the longitudinal associations between physical

362 activity, sedentary behavior and overweight/obesity ideally using objective measurements.

363

364 Conflict of interest

365 The authors declare no conflict of interest.

366

367 Supplementary information is available at the International Journal of Obesity's website 368

369 


\section{References}

371 1. Bouchard C, Blair SN, Haskell WL. Why Study Physical Activity and Health? In: Bouchard

372 C, Blair SN, Haskell WL (eds). Physical Activity and Health. Human Kinetics: Champaign, IL, 2007, 373 p. 12.

374 2. Physical Activity Guidelines Advisory Committee. Physical Activity Guidelines Advisory

375 Committee Report 2008. Washington, DC: U.S. Department of Health and Human Services, 2008.

3763 Fogelholm M, Kukkonen-Harjula K. Does physical activity prevent weight gain--a systematic 377 review. Obes Rev 2000;1(2):95-111.

378 4. Besson H, Ekelund U, Luan J, May AM, Sharp S, Travier N, et al. A cross-sectional analysis 379 of physical activity and obesity indicators in European participants of the EPIC-PANACEA study. Int $380 \quad J$ Obes (Lond) 2009;33(4):497-506.

381 5. Lee CM, Huxley RR, Wildman RP, Woodward M. Indices of abdominal obesity are better 382 discriminators of cardiovascular risk factors than BMI: a meta-analysis. J Clin Epidemiol $383 \quad$ 2008;61(7):646-53.

384 6. Czernichow S, Kengne AP, Stamatakis E, Hamer M, Batty GD. Body mass index, waist

385

386

387 circumference and waist-hip ratio: which is the better discriminator of cardiovascular disease mortality risk?: evidence from an individual-participant meta-analysis of 82864 participants from nine cohort studies. Obes Rev 2011;12(9):680-7.

7. Petursson H, Sigurdsson JA, Bengtsson C, Nilsen TI, Getz L. Body configuration as a predictor of mortality: comparison of five anthropometric measures in a 12 year follow-up of the Norwegian HUNT 2 study. PLoS One 2011;6(10):e26621.

8. Fowke JH, Matthews CE. PSA and body composition by dual X-ray absorptiometry (DXA) in NHANES. Prostate 2010;70(2):120-5.

9. Healy GN, Wijndaele K, Dunstan DW, Shaw JE, Salmon J, Zimmet PZ, et al. Objectively measured sedentary time, physical activity, and metabolic risk: the Australian Diabetes, Obesity and Lifestyle Study (AusDiab). Diabetes Care 2008;31(2):369-71.

10. Ekelund U, Griffin SJ, Wareham NJ. Physical activity and metabolic risk in individuals with a family history of type 2 diabetes. Diabetes Care 2007;30(2):337-42. 

physical activity and sedentary time of breast cancer survivors, and associations with adiposity:

400

401

402

403

404

405

406

407

408

409

410

411

412

413

414

415

416

417

418

419

420

421

422

423

424 findings from NHANES (2003-2006). Cancer Causes Control 2010;21(2):283-8.

12. Stamatakis E, Davis M, Stathi A, Hamer M. Associations between multiple indicators of objectively-measured and self-reported sedentary behaviour and cardiometabolic risk in older adults. Prev Med 2012;54(1):82-7.

13. Stamatakis E, Hamer M, Tilling K, Lawlor DA. Sedentary time in relation to cardio-metabolic risk factors: differential associations for self-report vs accelerometry in working age adults. Int $J$ Epidemiol 2012.

14. O'Donovan G, Hillsdon M, Ukoumunne OC, Stamatakis E, Hamer M. Objectively measured physical activity, cardiorespiratory fitness and cardiometabolic risk factors in the Health Survey for England. Prev Med 2013;57(3):201-5.

15. Strath SJ, Holleman RG, Ronis DL, Swartz AM, Richardson CR. Objective physical activity accumulation in bouts and nonbouts and relation to markers of obesity in US adults. Prev Chronic Dis 2008;5(4):A131.

16. Healy GN, Matthews CE, Dunstan DW, Winkler EA, Owen N. Sedentary time and cardiometabolic biomarkers in US adults: NHANES 2003-06. Eur Heart J 2011;32(5):590-7.

17. Maher CA, Mire E, Harrington DM, Staiano AE, Katzmarzyk PT. The independent and combined associations of physical activity and sedentary behavior with obesity in adults: NHANES 2003-06. Obesity (Silver Spring) 2013;21(12):E730-7.

18. Wareham NJ, van Sluijs EM, Ekelund U. Physical activity and obesity prevention: a review of the current evidence. Proc Nutr Soc 2005;64(2):229-47.

19. Johnson CL, Paulose-Ram R, Ogden CL, Carroll MD, Kruszon-Moran D, Dohrmann SM, et al. National Health and Nutrition Examination Survey: Analytic guidelines, 1999-2010. Vital Health Stat 2013;2(161).

20. Melanson EL, Jr., Freedson PS. Validity of the Computer Science and Applications, Inc. (CSA) activity monitor. Med Sci Sports Exerc 1995;27(6):934-40. 

United States measured by accelerometer. Med Sci Sports Exerc 2008;40(1):181-8.

427

428

22. Matthews CE, Chen KY, Freedson PS, Buchowski MS, Beech BM, Pate RR, et al. Amount of time spent in sedentary behaviors in the United States, 2003-2004. Am J Epidemiol 2008;167(7):87581.

23. Matthews CE. Calibration of accelerometer output for adults. Med Sci Sports Exerc 2005;37(11 Suppl):S512-22.

24. Metzger JS, Catellier DJ, Evenson KR, Treuth MS, Rosamond WD, Siega-Riz AM. Patterns of objectively measured physical activity in the United States. Med Sci Sports Exerc 2008;40(4):6308.

25. National Health and Nutrition Examination Survey. National Health and Nutrition Examination Survey: Technical Documentation for the 1999-2004 Dual Energy X-Ray Absorptiometry (DXA) Multiple Imputation Data files 2008 [cited 2015 2015/06/12]. Available from: http://www.cdc.gov/Nchs/Nhanes/dxx/dxa.htm.

26. Folsom AR, Kushi LH, Anderson KE, Mink PJ, Olson JE, Hong CP, et al. Associations of general and abdominal obesity with multiple health outcomes in older women: the Iowa Women's Health Study. Arch Intern Med 2000;160(14):2117-28.

27. Peterson MD, Al Snih S, Stoddard J, Shekar A, Hurvitz EA. Obesity misclassification and the metabolic syndrome in adults with functional mobility impairments: Nutrition Examination Survey 2003-2006. Prev Med 2014;60:71-6.

28. National Health and Nutrition Examination Survey. National Health and Nutrition Examination Survey: Anthropometry Procedures Manual 2004 [cited 2015 2015/11/17]. Available from: http://www.cdc.gov/nchs/data/nhanes/nhanes 03 04/BM.pdf.

29. World Health Organization. Obesity: preventing and managing the global epidemic. Report of a WHO Consultation. Geneva: WHO, 2000.

30. World Health Organization. Waist Circumference and Waist-Hip Ratio: Report of a WHO Expert Consultation, Geneva, 8-11 December 2008. Geneva: WHO, 2008. 
31. Lean ME, Han TS, Morrison CE. Waist circumference as a measure for indicating need for

453

454

455

456

457

458

459

460

461

462

463

464

465

466

467

468

469

470

471

472

473

474

475

476

477

478

479

weight management. BMJ 1995;311(6998):158-61.

32. Browning LM, Hsieh SD, Ashwell M. A systematic review of waist-to-height ratio as a screening tool for the prediction of cardiovascular disease and diabetes: 0.5 could be a suitable global boundary value. Nutr Res Rev 2010;23(2):247-69.

33. Ashwell M. Plea for simplicity: use of waist-to-height ratio as a primary screening tool to assess cardiometabolic risk. Clinical Obesity 2012:no-no.

34. Wanner M, Probst-Hensch N, Kriemler S, Meier F, Autenrieth C, Martin BW. Validation of the long International Physical Activity Questionaire: influence of age and language region. Prev Med Reports 2016;3:250-6.

35. Wanner M, Martin B, Autenrieth C, Schaffner E, Meier F, Brombach C, et al. Associations between domains of physical activity, sitting time, and different measures of overweight and obesity. Prev Med Reports 2016;3:177-84.

36. Morris MS, Jacques PF. Total protein, animal protein and physical activity in relation to muscle mass in middle-aged and older Americans. Br J Nutr 2013;109(7):1294-303.

37. Seo DC, Li K. Leisure-time physical activity dose-response effects on obesity among US adults: results from the 1999-2006 National Health and Nutrition Examination Survey. J Epidemiol Community Health 2010;64(5):426-31.

38. Stamatakis E, Hirani V, Rennie K. Moderate-to-vigorous physical activity and sedentary behaviours in relation to body mass index-defined and waist circumference-defined obesity. Br J Nutr 2009;101(5):765-73.

39. Monda KL, Adair LS, Zhai F, Popkin BM. Longitudinal relationships between occupational and domestic physical activity patterns and body weight in China. Eur J Clin Nutr 2008;62(11):131825.

40. Wanner M, Gotschi T, Martin-Diener E, Kahlmeier S, Martin BW. Active transport, physical activity, and body weight in adults: a systematic review. Am J Prev Med 2012;42(5):493-502.

41. Bauman AE, Reis RS, Sallis JF, Wells JC, Loos RJ, Martin BW. Correlates of physical activity: why are some people physically active and others not? Lancet 2012;380(9838):258-71. 
480 42. Pedisic Z, Grunseit A, Ding D, Chau JY, Banks E, Stamatakis E, et al. High sitting time or 481 obesity: Which came first? Bidirectional association in a longitudinal study of 31,787 Australian 482 adults. Obesity (Silver Spring) 2014;22(10):2126-30.

483 43. Pulsford RM, Stamatakis E, Britton AR, Brunner EJ, Hillsdon MM. Sitting behavior and 484 obesity: evidence from the Whitehall II study. Am J Prev Med 2013;44(2):132-8.

485 44. Ekelund U, Brage S, Besson H, Sharp S, Wareham NJ. Time spent being sedentary and weight 486 gain in healthy adults: reverse or bidirectional causality? Am J Clin Nutr 2008;88(3):612-7.

487

488 
Table 1. Characteristics of participants, by sex and weight status according to percent body fat,

\begin{tabular}{|c|c|c|c|c|}
\hline & \multicolumn{2}{|c|}{ Males } & \multicolumn{2}{|c|}{ Females } \\
\hline & $\begin{array}{l}\text { normal weight } \\
(<25 \% \text { body } \\
\text { fat })\end{array}$ & $\begin{array}{l}\text { overweight/obe } \\
\text { se }(\geq 25 \% \text { body } \\
\text { fat) }\end{array}$ & $\begin{array}{l}\text { normal weight } \\
(<35 \% \text { body } \\
\text { fat })\end{array}$ & $\begin{array}{l}\text { overweight/obese } \\
\text { ( } \geq 35 \% \text { body fat) }\end{array}$ \\
\hline $\mathrm{N}$ (unweighted) & 771 & 1689 & 509 & 1825 \\
\hline Proportion [\%] & 16.3 & 35.2 & 10.6 & 38.1 \\
\hline Age [years], mean (SE) & $37.4(0.7)$ & $45.9(0.5)$ & $(0.6)$ & $5.9(0.5)$ \\
\hline $\begin{array}{l}\text { Ethnicity [\%] } \\
\text { Non-Hispanic White } \\
\text { Non-Hispanic Black } \\
\text { Mexican American } \\
\text { Other }\end{array}$ & $\begin{array}{r}69.9 \\
13.6 \\
7.6 \\
8.9\end{array}$ & $\begin{array}{r}75.8 \\
8.1 \\
9.5 \\
6.6\end{array}$ & $\begin{array}{r}75.6 \\
7.6 \\
6.3 \\
10.5\end{array}$ & $\begin{array}{r}73.0 \\
11.5 \\
7.6 \\
7.9\end{array}$ \\
\hline $\begin{array}{l}\text { Marital status [\%] } \\
\text { Married/living together } \\
\text { Single (widowed, separated, } \\
\text { divorced) } \\
\text { Missing information }\end{array}$ & $\begin{array}{r}61.0 \\
39.0 \\
0.0\end{array}$ & $\begin{array}{r}73.5 \\
26.3 \\
0.2\end{array}$ & $\begin{array}{r}61.4 \\
38.3 \\
0.3\end{array}$ & $\begin{array}{r}65.6 \\
34.4 \\
0.0\end{array}$ \\
\hline $\begin{array}{l}\text { Poverty index ratio [\%] } \\
\text { Below poverty } \\
\text { At or above poverty } \\
\text { Missing information }\end{array}$ & $\begin{array}{l}.6 \\
.6\end{array}$ & $\begin{array}{r}7.7 \\
88.8 \\
3.5\end{array}$ & $\begin{array}{r}9.2 \\
87.4 \\
3.4\end{array}$ & $\begin{array}{r}8.9 \\
87.7 \\
3.4\end{array}$ \\
\hline $\begin{array}{l}\text { Education [\%] } \\
\text { High school or lower } \\
\text { Post secondary (college or higher) } \\
\text { Missing information }\end{array}$ & $\begin{array}{r}32.8 \\
61.4 \\
5.8\end{array}$ & $\begin{array}{r}39.3 \\
59.4 \\
1.3\end{array}$ & $\begin{array}{r}24.5 \\
70.3 \\
5.1\end{array}$ & $\begin{array}{r}38.6 \\
59.7 \\
1.7\end{array}$ \\
\hline Total body fat [\%], mean (SE) & $21.0(0.2)$ & $30.8(0.2)$ & $30.9(0.2)$ & $42.7(0.2)$ \\
\hline Android body fat [\%], mean (SE) & $21.7(0.3)$ & $35.8(0.2)$ & $27.2(0.4)$ & $42.2(0.2)$ \\
\hline Gynoid body fat [\%], mean (SE) & $21.8(0.2)$ & $29.8(0.2)$ & $36.1(0.3)$ & $44.2(0.2)$ \\
\hline Body Mass Index $\left[\mathrm{kg} / \mathrm{m}^{2}\right]$, mean (SE) & $23.8(0.1)$ & $30.0(0.2)$ & $21.9(0.1)$ & $30.4(0.3)$ \\
\hline Waist circumference $[\mathrm{cm}]$, mean $(\mathrm{SE})$ & $86.5(0.5)$ & $106.0(0.5)$ & $78.3(0.3)$ & $98.5(0.6)$ \\
\hline Waist-to-height ratio, mean (SE) & $0.49(0.003)$ & $0.60(0.003)$ & $0.48(0.002)$ & $0.61(0.004)$ \\
\hline $\begin{array}{l}\text { BMI category [\%] } \\
\text { Normal/underweight } \\
\text { Overweight } \\
\text { Obese } \\
\text { Missing information }\end{array}$ & $\begin{array}{r}68.3 \\
29.9 \\
1.5 \\
0.3\end{array}$ & $\begin{array}{r}10.8 \\
46.2 \\
42.7 \\
0.4\end{array}$ & $\begin{array}{r}92.5 \\
7.4 \\
0.1 \\
0.0\end{array}$ & $\begin{array}{r}22.0 \\
34.8 \\
42.7 \\
0.4\end{array}$ \\
\hline
\end{tabular}




\begin{tabular}{|c|c|c|c|c|}
\hline \multicolumn{5}{|l|}{ Waist circumference category [\%] } \\
\hline Normal weight & 82.8 & 14.0 & 62.4 & 7.4 \\
\hline Overweight & 13.2 & 26.4 & 30.1 & 16.1 \\
\hline Obese & 2.7 & 57.8 & 6.3 & 75.7 \\
\hline Missing information & 1.4 & 1.8 & 1.3 & 0.8 \\
\hline \multicolumn{5}{|l|}{ Waist-to-height ratio category [\%] } \\
\hline Normal weight & 60.8 & 3.8 & 71.9 & 8.2 \\
\hline Overweight & 37.4 & 51.9 & 26.2 & 46.0 \\
\hline Obese & 0.2 & 42.5 & 0.6 & 45.0 \\
\hline Missing information & 1.6 & 1.8 & 1.3 & 0.8 \\
\hline \multicolumn{5}{|l|}{$\begin{array}{l}\text { Objectively measured physical activity, } \\
\text { mean (SE) }\end{array}$} \\
\hline Wear time [hours/day] & $\begin{array}{r}14.6(0.09) \\
425(6)\end{array}$ & $\begin{array}{r}14.5(0.06) \\
346(5)\end{array}$ & $\begin{array}{r}14.2(0.11) \\
361(7)\end{array}$ & $\begin{array}{r}2(0.06) \\
290(4)\end{array}$ \\
\hline Mean total [counts/min] & $\begin{array}{r}425(6) \\
420(1.2)\end{array}$ & $\begin{array}{r}540(3) \\
28.2(0.8)\end{array}$ & & $\begin{array}{r}290(4) \\
16.7(0.6)\end{array}$ \\
\hline \multirow{2}{*}{\multicolumn{5}{|c|}{ Self-reported physical activity, mean }} \\
\hline & & & & \\
\hline Total leisure-time $[\mathrm{min} / \mathrm{d}]$ & $199.7(27.2)$ & $142.9(7.8)$ & $148.3(14.5)$ & $103.2(5.3)$ \\
\hline Moderate leisure-time [min/d] & $126.5(12.9)$ & $57.8(5.0)$ & $118.8(13.2)$ & $44.0(3.7)$ \\
\hline \multicolumn{5}{|l|}{ Vigorous leisure-time [min/d] } \\
\hline $\begin{array}{l}\text { Objectively measured sedentary } \\
\text { behavior [hours/day] }\end{array}$ & .08) & $8.2(0.07)$ & $7.7(0.11)$ & $8.0(0.06)$ \\
\hline Total energy intake [kcal], mean (SE) & $(42)$ & $2539(27)$ & $1914(44)$ & $1790(17)$ \\
\hline \multicolumn{5}{|l|}{ Smoking status [\%] } \\
\hline Current non-smoker & & 76.6 & 74.3 & 82.4 \\
\hline Current smoker & 31.5 & 23.4 & 25.7 & 17.6 \\
\hline \multicolumn{5}{|l|}{ Alcohol intake [\%] } \\
\hline No drinks & 30.6 & 26.8 & 28.9 & 36.0 \\
\hline$\leq 1$ drink/week & 19.9 & 30.1 & 36.4 & 42.0 \\
\hline$>1$ drink/week & 49.5 & 43.1 & 34.7 & 22.0 \\
\hline \multicolumn{5}{|l|}{ Health status [\%] } \\
\hline Excellent or very good & 57.4 & 46.1 & 65.3 & 42.5 \\
\hline Good & 30.3 & 37.1 & 22.0 & 38.6 \\
\hline Not so good or bad & 8.2 & 13.8 & 9.2 & 14.8 \\
\hline Missing information & 4.2 & 3.0 & 3.4 & 4.1 \\
\hline
\end{tabular}


Table 2. Associations between different intensities of objectively measured physical activity, sitting time and body weight (ordered logistic regressions)

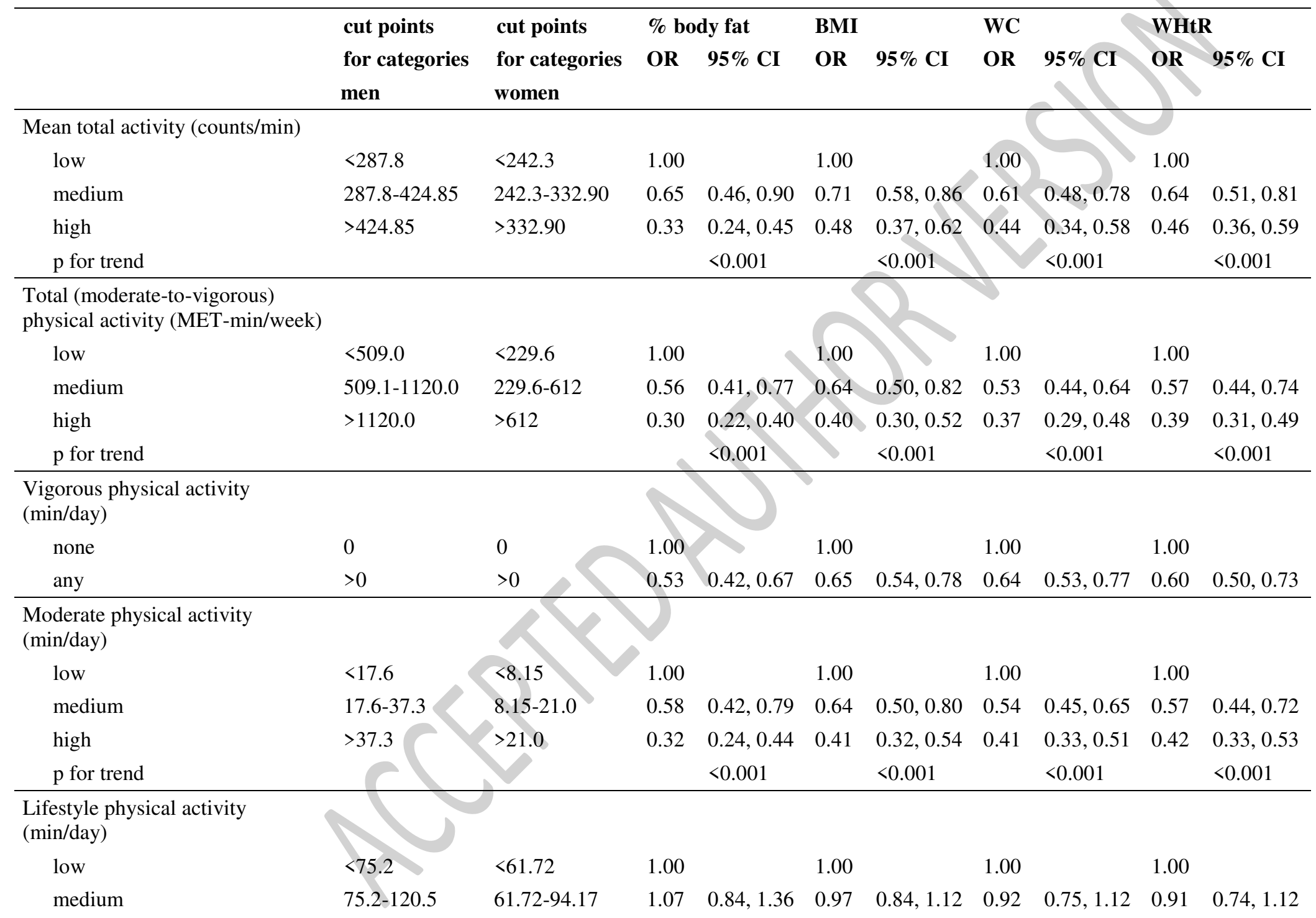




\begin{tabular}{|c|c|c|c|c|c|c|c|c|c|c|}
\hline $\begin{array}{l}\text { high } \\
\mathrm{p} \text { for trend }\end{array}$ & $>120.5$ & & 0.80 & $\begin{array}{l}0.60,1.07 \\
0.057\end{array}$ & 0.93 & $\begin{array}{l}0.73,1.19 \\
0.837\end{array}$ & 0.84 & $\begin{array}{l}0.64,1.11 \\
0.465\end{array}$ & 0.85 & $\begin{array}{l}0.66,1.10 \\
0.455\end{array}$ \\
\hline \multicolumn{11}{|c|}{ Light activities (min/day) } \\
\hline low & $<227.2$ & $<244.4$ & 1.00 & & 1.00 & & 1.00 & & 1. & \\
\hline medium & $227.2-283.0$ & $244.5-297.84$ & 0.91 & $0.70,1.19$ & 1.02 & $0.84,1.25$ & 0.89 & $0.71,1.12$ & 0.87 & $0.70,1.09$ \\
\hline high & $>283.0$ & $>297.84$ & 0.98 & $0.74,1.32$ & 1.14 & $0.92,1.41$ & 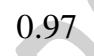 & $0.76,1.24$ & 0.88 & $0.73,1.05$ \\
\hline $\mathrm{p}$ for trend & & & & 0.736 & & 0.443 & & 0.569 & & 0.318 \\
\hline \multicolumn{11}{|c|}{ Sitting time (min/day) } \\
\hline low & $<420.1$ & $<427.6$ & 1.00 & & 1.00 & & 1. & & 1.00 & \\
\hline medium & $420.1-527.6$ & $427.6-519.1$ & 1.13 & $0.86,1.47$ & 0.94 & $0.77,1$. & 0.93 & $0.72,1.18$ & 0.99 & $0.81,1.22$ \\
\hline high & $>527.6$ & $>519.1$ & 1.17 & $0.92,1.49$ & 1.04 & $0.87,1.23$ & 1.13 & $0.87,1.48$ & 1.18 & $0.94,1.47$ \\
\hline$p$ for trend & & & & 0.445 & & 0.463 & & 0.162 & & 0.113 \\
\hline
\end{tabular}

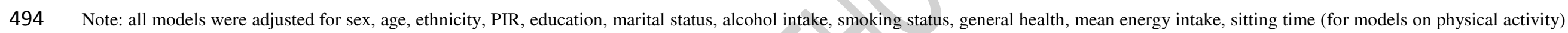
and moderate-to-vigorous physical activity (models on sitting time), respectively.

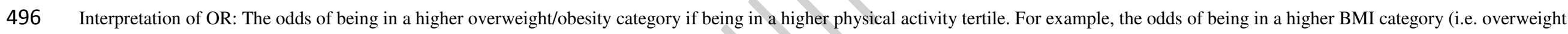

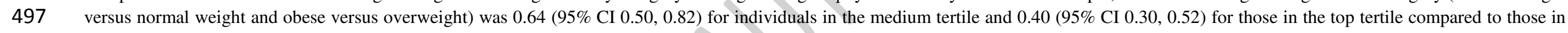
498 the bottom tertile of moderate-to-vigorous physical activity. 
Table 3. Associations between different domains and intensities of self-reported physical activity and body weight (ordered logistic regressions)

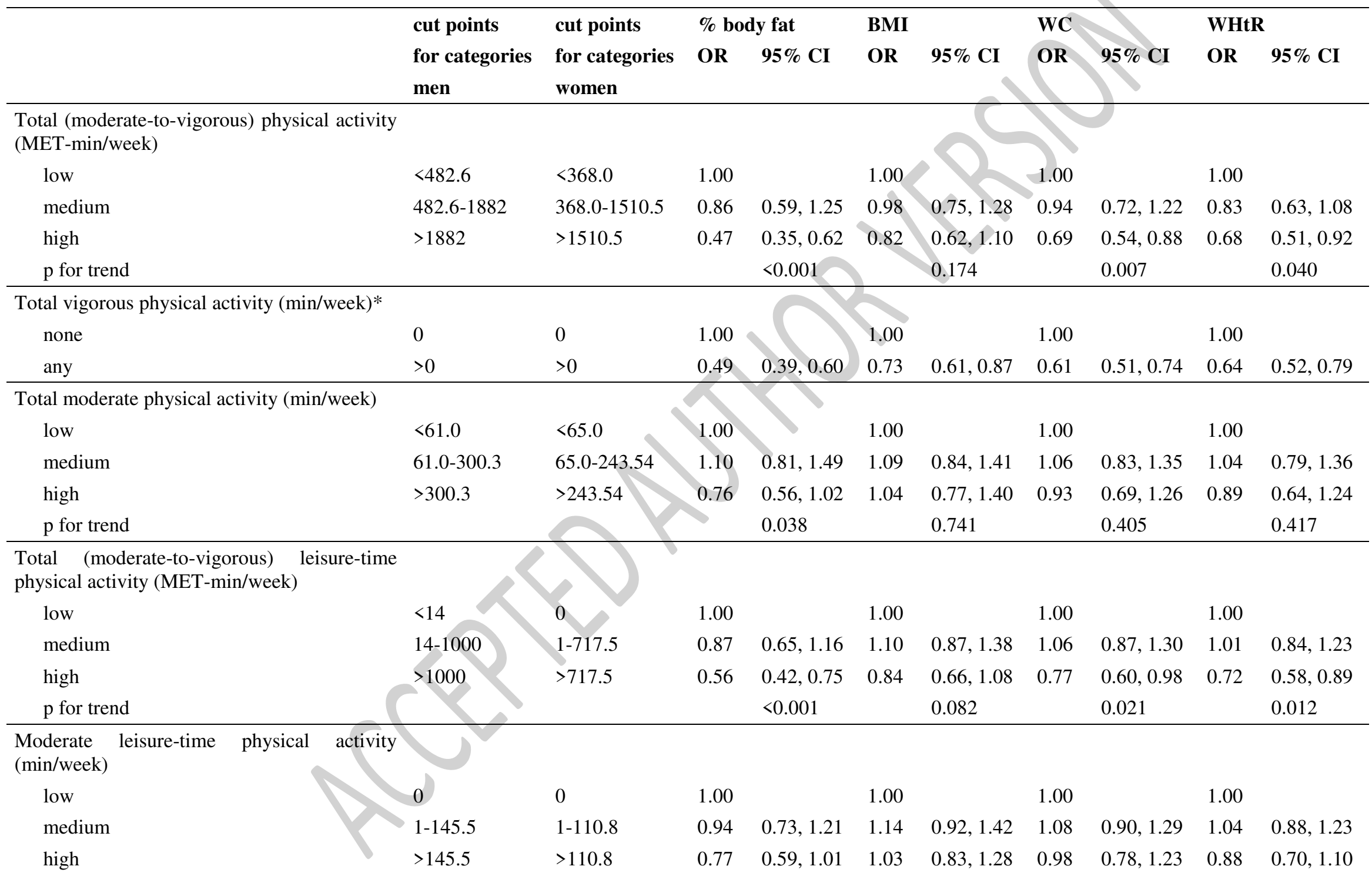




\begin{tabular}{|c|c|c|c|c|c|c|c|c|c|c|}
\hline $\mathrm{p}$ for trend & & & & 0.160 & & 0.444 & & 0.578 & & 0.468 \\
\hline \multicolumn{11}{|c|}{ Transport physical activity (MET-min/week) } \\
\hline none & 0 & 0 & 1.00 & & 1.00 & & 1.00 & & 1.00 & \\
\hline any & $>0$ & $>0$ & 0.78 & $0.64,0.94$ & 0.74 & $0.60,0.91$ & 0.77 & $0.64,0.92$ & 0.78 & $0.63,0.96$ \\
\hline \multicolumn{11}{|c|}{ Household physical activity (MET-min/week) } \\
\hline low & $<5$ & 0 & 1.00 & & 1.00 & & 1.00 & & 1.00 & \\
\hline medium & $5-380$ & $1-380$ & 1.30 & $1.03,1.64$ & 1.28 & $1.05,1.57$ & 1.35 & $1.11,1.64$ & 1.31 & $1.08,1.59$ \\
\hline high & $>380$ & $>380$ & 1.13 & $0.86,1.49$ & 1.50 & $1.20,1.88$ & 1.43 & $1.11,1.84$ & 1.35 & $1.07,1.70$ \\
\hline $\mathrm{p}$ for trend & & & & 0.070 & & 0.004 & & 0.008 & & 0.020 \\
\hline
\end{tabular}

*total vigorous physical activity is equal to leisure-time vigorous physical activity, as only the domain leisure-time includes vigorous activities

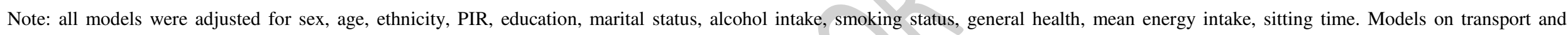
household physical activity were adjusted for total leisure-time physical activity in addition.

Interpretation of OR: The odds of being in a higher overweight/obesity category if being in a higher physical activity tertile. For example, the odds of being in a higher BMI category (i.e. overweight

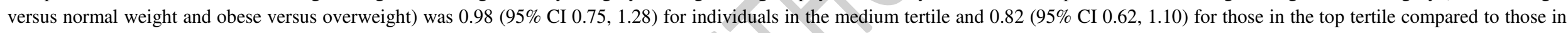
the bottom tertile of total moderate-to-vigorous physical activity. 


\section{4 different measures of overweight/obesity}

$\mathrm{OR}$

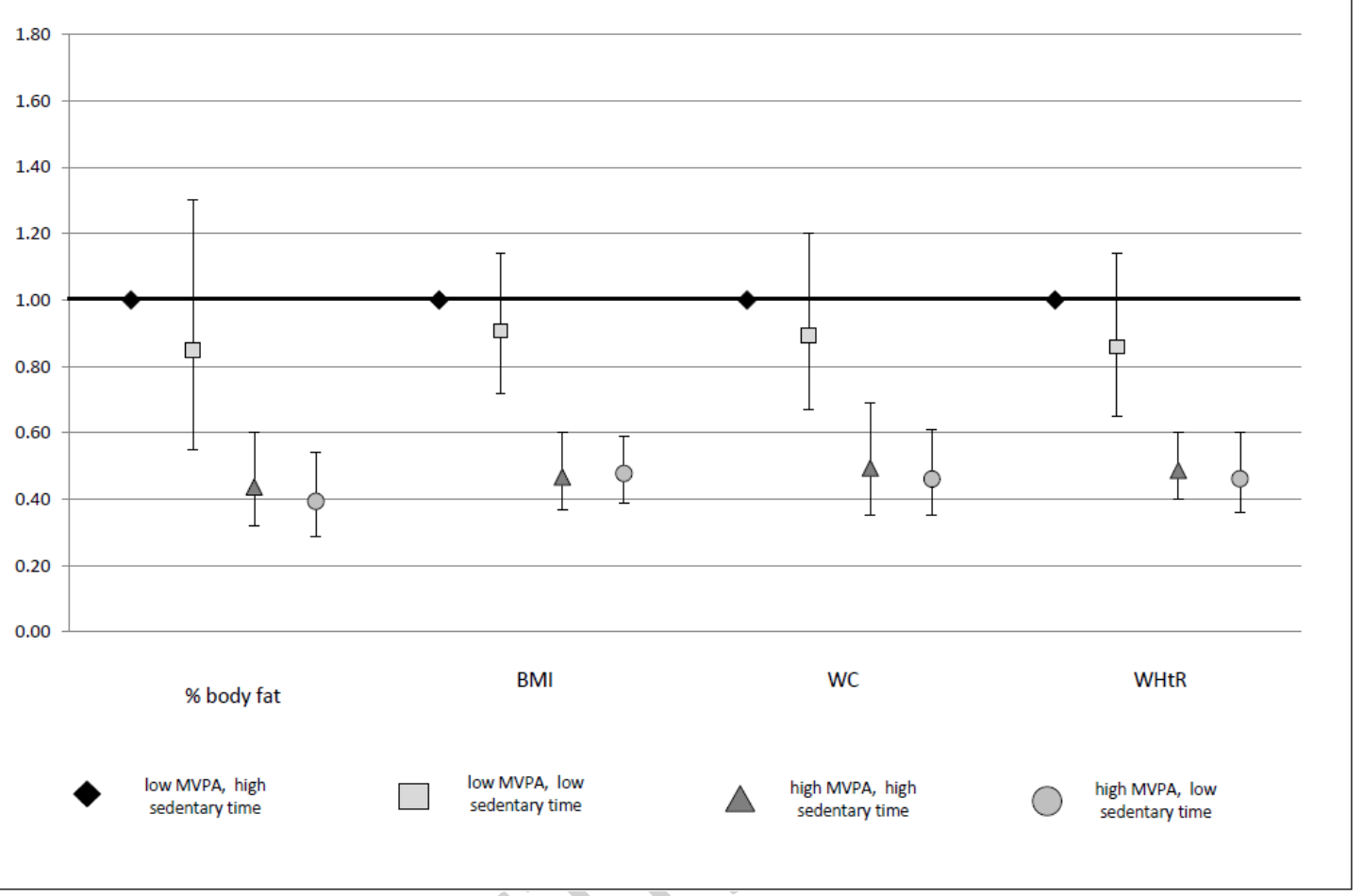

515

516 Abbreviations: BMI, body mass index; MVPA, moderate-to-vigorous physical activity; OR, odds ratio; WC, waist circumference; WHtR, waist-to-height ratio 
Supplementary Information Table 1. Associations between objective and self-reported physical activity and percent body fat (total, android, gynoid) based on linear regression, by sex, NHANES 2003-06

\begin{tabular}{|c|c|c|c|c|c|c|c|c|c|c|c|c|c|c|}
\hline & \multirow{2}{*}{\multicolumn{2}{|c|}{\begin{tabular}{|l|} 
\% total body fat \\
Total
\end{tabular}}} & \multicolumn{6}{|c|}{ \% body fat (android) } & \multicolumn{6}{|c|}{ \% body fat (gynoid) } \\
\hline & & & \multicolumn{2}{|l|}{ Total } & \multicolumn{2}{|l|}{ Men } & \multicolumn{2}{|c|}{ Women } & \multicolumn{2}{|l|}{ Total } & \multicolumn{2}{|l|}{ Men } & \multicolumn{2}{|c|}{ Women } \\
\hline & beta & $95 \% \mathrm{CI}$ & beta & $95 \% \mathrm{CI}$ & beta & $95 \% \mathrm{CI}$ & beta & $95 \% \mathrm{CI}$ & beta & $95 \% \mathrm{CI}$ & beta & $95 \% \mathrm{CI}$ & beta & $95 \% \mathrm{CI}$ \\
\hline \multicolumn{15}{|l|}{ Objective physical activity } \\
\hline Mean total PA (10 counts/min) & -0.119 & $-0.139,-0.098$ & -0.143 & $-0.173,-0.113$ & -0.098 & $-0.127,-0.068$ & -0.204 & $-0.266,-0.143$ & -0.106 & $-0.127,-0.084$ & -0.090 & $-0.109,-0.071$ & -0.127 & $-0.164,-0.089$ \\
\hline $\begin{array}{l}\text { Total MVPA } \quad(100 \quad \text { MET- } \\
\text { min/week })\end{array}$ & -0.211 & $-0.247,-0.174$ & -0.246 & $-0.300,-0.192$ & -0.157 & $-0.206,-0.108$ & -0.400 & $-0.537,-0.262$ & -0.188 & $-0.223,-0.152$ & -0.145 & $-0.178,-0.112$ & -0.266 & $-0.342,-0.189$ \\
\hline Vigorous PA (10 min/day) & -2.454 & $-3.124,-1.784$ & -3.111 & $-4.297,-1.924$ & -1.753 & $-3.270,-0.237$ & -4.797 & $-6.629,-2.965$ & -2.053 & $-2.594,-1.511$ & -1.282 & $-1.930,-0.634$ & -3.073 & $-4.372,-1.775$ \\
\hline Moderate PA (10 min/day) & -0.596 & $-0.718,-0.474$ & -0.675 & $-0.850,-0.499$ & -0.460 & $-0.620,-0.299$ & -1.055 & $-1.489,-0.621$ & -0.542 & $-0.668,-0.416$ & -0.450 & $-0.567,-0.333$ & -0.712 & $-0.991,-0.434$ \\
\hline Lifestyle PA (10 min/day) & -0.156 & $-0.207,-0.104$ & -0.225 & $-0.327,-0.124$ & -0.201 & $-0.302,-0.099$ & -0.263 & $-0.416,-0.111$ & -0.161 & $-0.215,-0.107$ & -0.199 & $-0.268,-0.130$ & -0.111 & $-0.185,-0.036$ \\
\hline Light PA (10 min/day) & -0.046 & $-0.085,-0.006$ & -0.080 & $-0.156,-0.004$ & -0.097 & $-0.208,0.014$ & -0.049 & $-0.168,0.070$ & -0.037 & $-0.096,0.023$ & -0.053 & $-0.134,0.028$ & -0.019 & $-0.098,0.061$ \\
\hline $\begin{array}{l}\text { Mean sedentary time } \\
\text { hour/day) }\end{array}$ & 0.159 & $0.019,0.299$ & 0.101 & $-0.120,0.321$ & 0.082 & $-0.182,0.346$ & 0.121 & $-0.174,0.416$ & 0.074 & $-0.068,0.216$ & 0.062 & $-0.114,0.239$ & 0.098 & $-0.116,0.312$ \\
\hline \multicolumn{15}{|l|}{ Self-reported physical activity } \\
\hline Total PA (100 MET-min/week) & -0.021 & $-0.034,-0.009$ & -0.025 & $-0.047,-0.003$ & -0.015 & $-0.039,0.008$ & -0.041 & $-0.068,-0.015$ & -0.023 & $-0.039,-0.007$ & -0.016 & $-0.034,0.001$ & -0.035 & $-0.053,-0.016$ \\
\hline $\begin{array}{l}\text { Total vigorous PA } \\
\text { min/day)* }\end{array}$ & -0.406 & $-0.518,-0.294$ & -0.524 & $-0.759,-0.289$ & -0.391 & $-0.652,-0.131$ & -0.661 & $-0.926,-0.397$ & -0.421 & $-0.538,-0.303$ & -0.337 & $-0.472,-0.202$ & -0.515 & $-0.677,-0.352$ \\
\hline Total moderate PA (10 min/day) & -0.023 & $-0.062,0.015$ & -0.019 & $-0.085,0.048$ & 0.0002 & $-0.079,0.080$ & -0.064 & $-0.148,0.020$ & -0.027 & $-0.082,0.028$ & -0.015 & $-0.075,0.046$ & -0.057 & $-0.127,0.012$ \\
\hline $\begin{array}{l}\text { Total (MVPA) LTPA } \quad(100 \\
\text { MET-min/week) }\end{array}$ & -0.044 & $-0.060,-0.028$ & -0.056 & $-0.084,-0.028$ & -0.033 & $-0.064,-0.001$ & -0.093 & $-0.125,-0.061$ & -0.045 & $-0.064,-0.027$ & -0.034 & $-0.055,-0.013$ & -0.064 & $-0.087,-0.040$ \\
\hline Moderate LTPA (10 min/day) & -0.048 & $-0.129,0.032$ & -0.056 & $-0.183,0.071$ & 0.004 & $-0.140,0.147$ & -0.203 & $-0.358,-0.048$ & -0.050 & $-0.145,0.045$ & -0.033 & $-0.130,0.064$ & -0.092 & $-0.244,0.059$ \\
\hline $\begin{array}{l}\begin{array}{l}\text { Transport } \\
\text { min/week })\end{array} \\
\text { PA } \quad(100 \quad \text { MET- } \\
\end{array}$ & -0.021 & $-0.048,0.006$ & -0.037 & $-0.099,0.026$ & -0.033 & $-0.114,0.049$ & -0.081 & $-0.169,0.007$ & -0.030 & $-0.072,0.012$ & -0.026 & $-0.077,0.025$ & -0.059 & $-0.140,0.022$ \\
\hline $\begin{array}{l}\text { Household PA } \quad(100 \text { MET- } \\
\text { min/week })\end{array}$ & 0.007 & $-0.007,0.020$ & 0.016 & $-0.007,0.039$ & 0.014 & $-0.012,0.039$ & 0.017 & $-0.017,0.052$ & 0.006 & $-0.011,0.024$ & 0.011 & $-0.007,0.029$ & -0.002 & $-0.028,0.024$ \\
\hline
\end{tabular}

*total vigorous physical activity is equal to leisure-time vigorous physical activity, as only the domain leisure-time includes vigorous activities 
Note: all models were adjusted for sex, age, ethnicity, PIR, education, marital status, alcohol intake, smoking status, general health, mean energy intake, sitting time based on accelerometer data (for models on physical activity) and moderate-to-vigorous physical activity (models with sedentary time), respectively. LTPA, leisure-time physical activity; MET, metabolic equivalent; MVPA, moderate-to-vigorous physical activity; PA, physical activity 